Pacific

Journal of

Mathematics

ON THE DEFORMATION QUANTIZATION OF COADJOINT ORBITS OF SEMISIMPLE GROUPS

R. Fioresi ANd M.A. Lledó 


\title{
ON THE DEFORMATION QUANTIZATION OF COADJOINT ORBITS OF SEMISIMPLE GROUPS
}

\author{
R. Fioresi And M.A. Lledó
}

To the memory of Moshe Flato

\begin{abstract}
In this paper we consider the problem of deformation quantization of the algebra of polynomial functions on coadjoint orbits of semisimple Lie groups. The deformation of an orbit is realized by taking the quotient of the universal enveloping algebra of the Lie algebra of the given Lie group, by a suitable ideal. A comparison with geometric quantization in the case of $\mathrm{SU}(2)$ is done, where both methods agree.
\end{abstract}

\section{Introduction.}

A system in classical mechanics is given by a symplectic manifold $X$ which we call phase space and a function on $X, H$, which we call Hamiltonian. The points in $X$ represent possible states of the system, the commutative algebra $C^{\infty}(X)$ is the set of classical observables, corresponding to possible measurements on the system, and the integral curves of the Hamiltonian vector field $X_{H}$ represent the time evolution of the classical system.

A quantization of the classical system $X$ has three ingredients $[\mathbf{B e}]$ :

1. A family of noncommutative complex algebras $\mathbf{A}_{h}$ depending on a real parameter $h$, which we will identify with Planck's constant, satisfying

$$
\mathbf{A}_{h} \mapsto \mathbf{A}=C^{\infty}(X)^{\mathbf{C}} \quad \text { when } \quad h \mapsto 0,
$$

or a suitable subalgebra of $C^{\infty}(X)^{\mathbf{C}}$ determined by physical requirements, but enough to separate the points of $X . C^{\infty}(X)^{\mathbf{C}}$ denotes the complexification of $C^{\infty}(X)$.

2. A family of linear maps $Q_{h}: \mathbf{A} \mapsto \mathbf{A}_{h}$, called the quantization maps satisfying

$$
\frac{Q_{h}(F) *_{h} Q_{h}(G)-Q_{h}(G) *_{h} Q_{h}(F)}{h} \mapsto\{F, G\} \quad \text { when } \quad h \mapsto 0,
$$

where $\{$,$\} is the Poisson bracket in \mathbf{A}$ (extended by linearity).

3. A representation of $\mathbf{A}_{h}$ on a Hilbert space $\mathcal{H}_{X}, R: \mathbf{A}_{h} \mapsto \operatorname{End}\left(\mathcal{H}_{X}\right)$. The real functions in $\mathbf{A}_{h}$ (belonging to $C^{\infty}(X)$ ) are mapped into hermitian operators. 
The elements of $\mathbf{A}_{h}$ are the quantum observables and the rays in $\mathcal{H}_{X}$ are the states of the quantum system. Not every possible realization of $\mathbf{A}_{h}$ on a Hilbert space $\mathcal{H}_{X}$ satisfies the physical requirements for the quantum system, since the set of rays of $\mathcal{H}_{X}$ should be in one to one correspondence with the quantum physical states. So further requirements should be imposed on $\mathcal{H}_{X}$.

A first step to find a quantization of a physical system is the construction of a formal deformation of the Poisson algebra classical observables [BFFLS]. In general, formal deformations do not present a closed solution to the quantization problem. One needs to see if it is possible to specialize the deformation to an interval of values of the formal parameter $h$ (including 0 , so the limit $h \mapsto 0$ is smooth), besides constructing the Hilbert space where this algebra is represented. Nevertheless having a formal deformation is a powerful technical tool in the process of quantization.

A first approach to this problem appears in [Be]. Berezin explicitly computes *-products for Kähler manifolds that are homogeneous spaces. His approach provides an explicit integral formula for a $*$-product where $\mathrm{h}$ is a real number. In [RCG] a geometric construction of Berezin's quantization is performed.

Later De Wilde and Lecomte $[\mathbf{D L}]$ and Fedosov $[\mathbf{F e}]$ separately, constructed and classified formal $*$-products on generic symplectic manifolds. Etingof and Kazhdan [EK] proved the existence of a formal deformation for another class of Poisson manifolds, the Poisson-Lie groups. Finally, Kontsevich $[\mathbf{K o}]$ proved the existence of an essentially unique formal $*$-product on general Poisson manifolds.

More recently Reshetekhin and Taktajan $[\mathbf{R T}]$, starting from Berezin's construction, were able to give an explicit integral formula for the formal *-product on Kähler manifolds.

It is our purpose to study the deformation quantization of coadjoint orbits of semisimple Lie groups. In $[\mathbf{A L M}]$ it has been proven that a covariant $*-$ product exists on the orbits of the coadjoint orbit that admit a polarization. We will consider the algebra of polynomials on coadjoint orbits. In the above mentioned works *-products are given on $C^{\infty}$ functions, however there is no guarantee that there is a subalgebra of functions that is closed under it. Instead, we will obtain both a formal deformation and a deformation for any real value of $h$ for the subalgebra of polynomial functions.

In $[\mathbf{K o}]$ Kontsevich briefly describes the algebra of polynomials over the dual of the Lie algebra (a Poisson manifold) as a special case of his general formula for $*$-product on Poisson manifolds (this special case was known long before $[\mathbf{G u}]$ ). He does not however consider the restriction of those polynomials to a coadjoint orbit submanifold and, as he points out later, the knowledge of $*$-product on a certain domain is far from giving knowledge 
of *-product on subdomains of it. The formulation of a star product on some coadjoint orbits using this deformation of the polynomial algebra was investigated in the series of works $[\mathbf{C G}],[\mathbf{A C G}]$ and $[\mathbf{H o}]$ (and references inside).

Our approach starts also from the fact that the universal enveloping algebra of a complex semisimple Lie algebra is the deformation quantization of the polynomial algebra on the dual Lie algebra. By quotienting by a suitable ideal we get a deformation quantization of the polynomial algebra on a regular coadjoint orbit. Using some known facts on real and complex orbits this gives us a deformation quantization on the regular orbits of compact semisimple Lie groups. No selection of ordering rule is needed for the proof, which means that we obtain a whole class of star products on the orbit. A proof of the analiticity of the deformation in the deformation parameter is provided here, and the convergence of the deformed product for polynomials on the orbit is obtained. More general cases, as regular orbits of non-compact Lie groups, involve some subtleties that are partially explored in Section 2. Further developments will be given in a subsequent paper. Also, the extension of the proof to non-regular (although still semisimple) orbits is non-trivial.

Our construction has the advantage that it is given in a coordinate independent way. Also the symmetries and its possible representations are better studied in this framework. The formal deformation is realized using a true deformation of the polynomials on the complex orbit. We obtain the deformation quantization as a non-commutative algebra depending on a formal parameter $h$ containing a subalgebra in which $h$ can be specialized to any real value.

Geometric quantization is another approach to the problem. The elements of the quantum system are constructed using the geometric elements of the classical system. (For an introduction to geometric quantization, see for example $[\mathbf{P u}]$ and references inside.) In the case when the phase space is $\mathbf{R}^{2 n}$, a comparison between both procedures, deformation and geometric quantization has been established $[\mathbf{G V}]$. Less trivial systems, as coadjoint orbits, have been the subject of geometric quantization. The guiding principle is the preservation of the symmetries of the classical system after the quantization. The idea of finding a unitary representation of the symmetry group naturally attached to the coadjoint orbit is known as the KirillovKostant orbit principle. The action of the group on the Hilbert space of the representation should be induced by the action of the group on the phase space as symplectomorphisms. The algebra of classical observables should be substituted by a non-commutative algebra and the group should act also naturally by conjugation on this algebra. 
The procedure we used in constructing the formal deformation, that is assigning an ideal in the enveloping algebra to the coadjoint orbit, makes the comparison with geometric quantization easier. In Section 4 we show that in the special case of $\mathrm{SU}(2)$ there is an isomorphism between our deformation quantization and the algebra of twisted differential operators that appears in geometric quantization.

The organization of the paper is as follows. In Section 2 we make a review of the algebraic properties of the coadjoint orbits on which our method of deformation is based. In Section 3 we prove the existence of the deformation and describe it explicitly in terms of a quotient of the enveloping algebra by an ideal. In Section 4 we make a comparison of our results with the results of geometric quantization for a particularly simple case, the coadjoint orbits of $\mathrm{SU}(2)$.

\section{Algebraic Structure of Coadjoint Orbits of Semisimple Lie Groups.}

Let $G_{R}$ be a real Lie group and $\mathcal{G}_{R}$ its Lie algebra. The coadjoint action of $G_{R}$ on $\mathcal{G}_{R}{ }^{*}$ is given by

$$
\left\langle\operatorname{Ad}^{*}(g) \lambda, Y\right\rangle=\left\langle\lambda, \operatorname{Ad}\left(g^{-1}\right) Y\right\rangle \quad \forall g \in G_{R}, \quad \lambda \in \mathcal{G}_{R}{ }^{*}, \quad Y \in \mathcal{G}_{R} .
$$

We will denote by $C_{G_{R}}(\lambda)$ (or simply $C_{\lambda}$ if $G_{R}$ can be suppressed without confusion) the orbit of the point $\lambda \in \mathcal{G}_{R}{ }^{*}$ under the coadjoint action of $G_{R}$.

Consider now the algebra of $C^{\infty}$ functions on $\mathcal{G}_{R}{ }^{*}, C^{\infty}\left(\mathcal{G}_{R}{ }^{*}\right)$. We can turn it into a Poisson algebra with the so called Lie-Poisson structure

$$
\left\{f_{1}, f_{2}\right\}(\lambda)=\left\langle\left[\left(d f_{1}\right)_{\lambda},\left(d f_{2}\right)_{\lambda}\right], \lambda\right\rangle, \quad f_{1}, f_{2} \in C^{\infty}\left(\mathcal{G}_{R}{ }^{*}\right), \quad \lambda \in \mathcal{G}_{R}{ }^{*} .
$$

If $f \in C^{\infty}\left(\mathcal{G}_{R}{ }^{*}\right),(d f)_{\lambda}$ is a map from $\mathcal{G}_{R}{ }^{*}$ to $\mathbf{R}$, so it can be regarded as an element of $\mathcal{G}_{R}$ and [, ] is the Lie bracket in $\mathcal{G}_{R}$. By writing the Poisson bracket in linear coordinates, it is clear that $\mathbf{R}\left[\mathcal{G}_{R}{ }^{*}\right]$, the ring of polynomials on $\mathcal{G}_{R}{ }^{*}$, is closed under the Poisson bracket.

The Hamiltonian vector fields define an integrable distribution on $\mathcal{G}_{R}{ }^{*}$ whose integral manifolds (the symplectic leaves) are precisely the orbits of the coadjoint action. So all the coadjoint orbits are symplectic manifolds with the symplectic structure inherited from the Poisson structure on $\mathcal{G}_{R}{ }^{*}$.

Let $G$ be a connected complex, semisimple Lie group and $\mathcal{G}$ its Lie algebra. We wish to describe the coadjoint orbits of different real forms of $G$. We can identify $\mathcal{G}$ and $\mathcal{G}^{*}$ by means of the Cartan-Killing form, so we will work with the adjoint action instead. We denote by $G_{R}$ an arbitrary real form of $G$, and $\mathcal{G}_{R}$ its Lie algebra.

We start with the adjoint orbits of the complex group $G$ itself. Let $Z_{s} \in \mathcal{G}_{R} \subset \mathcal{G}$ be a semisimple element. The orbit of $Z_{s}$ in $\mathcal{G}$ under $G$ will be denoted by $C_{G}\left(Z_{s}\right)$. It is well known that this orbit is a smooth 
complex algebraic variety defined over $\mathbf{R}[\mathbf{B o}]$. That means that the real form of $C_{G}\left(Z_{s}\right), C_{G}\left(Z_{s}\right)(\mathbf{R})=C_{G}\left(Z_{s}\right) \cap \mathcal{G}_{R}$ is a real algebraic variety. If $G_{R}$ is compact, $C_{G}\left(Z_{s}\right)(\mathbf{R})$ coincides with the real orbit $C_{G_{R}}\left(Z_{s}\right)$. In general $C_{G}\left(Z_{s}\right)(\mathbf{R})$ is the union of several real orbits $C_{G_{R}}\left(X_{i}\right), i \in I$ for some finite set of indices $I[\mathbf{V a 2}]$. Hence the real orbits are not always algebraic varieties. We will give one of such examples later. Still, the algebraic structure of the closely related manifold $C_{G}\left(Z_{s}\right)(\mathbf{R})$ will be useful for the quantization.

The algebra that we want to deform is the polynomial ring on the complex orbit. When $C_{G}\left(Z_{s}\right)(\mathbf{R})$ consists of one real orbit, the complex polynomial ring is the complexification of the polynomial ring on the real orbit. In this case, giving a formal deformation defined over $\mathbf{R}$ of the polynomial ring of the complex orbit is completely equivalent to give a formal deformation of the polynomial ring of the real orbit.

In general $I$ will have many elements. One can always consider the algebra of polynomials on $C_{G}\left(Z_{s}\right)(\mathbf{R})$ and restrict it to each of the connected components. The $*$-product we obtain can also be defined on the algebra of restricted polynomials without ambiguity, so we have a deformation of certain algebra of functions on the real orbit. Interesting subalgebras of the restricted polynomials that still separate the points of the real orbit could be found, being also closed under the $*$-product. We will see such kind of construction in an example.

We summarize now the classification of real coadjoint orbits [Va2], [Vo]. The easiest situation is when $G_{R}$ is a compact group. In this case the orbits are real algebraic varieties defined by the polynomials on $\mathcal{G}$, invariant with respect to the coadjoint action. These invariant polynomials (or Casimir polynomials) are in one to one correspondence with polynomials on the Cartan subalgebra that are invariant under the Weyl group. So every point in a Weyl chamber determines a value of the invariant polynomials, and hence, an adjoint orbit.

The general case is a refinement of this particular one. We will consider only orbits that contain a semisimple element $Z_{s} \in \mathcal{G}_{R}$. There are two special cases: The elliptic orbits, when the minimal polynomial of the element $Z_{e}$ has only purely imaginary eigenvalues, and the hyperbolic orbits, when the minimal polynomial of $Z_{h}$ has only real eigenvalues. The general case $Z_{s}=$ $Z_{h}+Z_{e}$ can be understood in terms of the special cases.

Let us denote by $U$ a compact real form of $G$ and $\mathcal{U}$ its Lie algebra, while $G_{0}$ and $\mathcal{G}_{0}$ denote a non-compact form and its Lie algebra. The involution $\theta$ : $\mathcal{G}_{0} \mapsto \mathcal{G}_{0}$ induces the Cartan decomposition $\mathcal{G}_{0}=\mathcal{L}_{0}+\mathcal{P}_{0}$, and $\mathcal{U}=\mathcal{L}_{0}+i \mathcal{P}_{0}$. $K$ is a maximal compact subgroup of $G_{0}$ with Lie algebra $\mathcal{L}_{0}$. We denote by $\mathcal{H}_{\mathcal{P}_{0}}$ the maximal abelian subalgebra of $\mathcal{P}_{0}$ and by $\mathcal{H}_{\mathcal{L}_{0}}$ a CSA of $\mathcal{L}_{0}$. $W\left(G_{0}, \mathcal{H}_{\mathcal{L}_{0}}\right)$ and $W\left(G_{0}, \mathcal{H}_{\mathcal{P}_{0}}\right)$ will denote the Weyl groups corresponding to 
the root systems of $K\left(W\left(G_{0}, \mathcal{H}_{\mathcal{L}_{0}}\right)\right)$ and the restricted root system of $G_{0}$ $\left(W\left(G_{0}, \mathcal{H}_{\mathcal{P}_{0}}\right)\right)$.

The set of hyperbolic orbits is in one to one correspondence with the set of orbits of $W\left(G_{0}, \mathcal{H}_{\mathcal{P}_{0}}\right)$ on $\mathcal{H}_{\mathcal{P}_{0}}$, while the set of elliptic orbits is in one to one correspondence with the set of orbits of $W\left(G_{0}, \mathcal{H}_{\mathcal{L}_{0}}\right)$ on $\mathcal{H}_{\mathcal{L}_{0}}$. In summary, each point in the Weyl chamber of the corresponding root system determines a unique semisimple orbit and vice versa.

Example 2.1 (Orbits of $S O(2,1)$ ). We want to show explicitly an example where the real form of the complex orbit is the union of two real orbits. The value of the invariant polynomials in this case doesn't completely determine a real orbit.

Consider the connected component containing the identity of the noncompact orthogonal group $\mathrm{SO}(2,1)=\left\{3 \times 3\right.$ real matrices $\left.\Lambda / \Lambda^{T} \eta \Lambda=\eta\right\}$, where

$$
\eta=\left(\begin{array}{ccc}
1 & 0 & 0 \\
0 & 1 & 0 \\
0 & 0 & -1
\end{array}\right)
$$

The Lie algebra $\operatorname{so}(2,1)$ is given by $\operatorname{so}(2,1)=\operatorname{span}\{G, \tilde{E}, \tilde{F}\}$, where

$$
G=\left(\begin{array}{ccc}
0 & 1 & 0 \\
-1 & 0 & 0 \\
0 & 0 & 0
\end{array}\right) \quad \tilde{E}=\left(\begin{array}{ccc}
0 & 0 & 0 \\
0 & 0 & 1 \\
0 & 1 & 0
\end{array}\right), \quad \tilde{F}=\left(\begin{array}{ccc}
0 & 0 & 1 \\
0 & 0 & 0 \\
1 & 0 & 0
\end{array}\right)
$$

with commutation relations

$$
[G, \tilde{E}]=\tilde{F}, \quad[G, \tilde{F}]=-\tilde{E}, \quad[\tilde{E}, \tilde{F}]=-G .
$$

The involutive automorphism associated to this non-compact form of so(3) is $\sigma(X)=\eta X \eta$ so the Cartan decomposition is given by $\mathcal{L}_{0}=\operatorname{span}\{G\}$ and $\mathcal{P}_{0}=\operatorname{span}\{\tilde{E}, \tilde{F}\} . \quad \mathcal{L}_{0}$ is the Lie algebra of $\mathrm{SO}(2)$, the maximal compact subgroup, which in this case is abelian.

The only Casimir polynomial is given in the coordinates $X=x \tilde{E}+y \tilde{F}+z G$ by $P(X)=x^{2}+y^{2}-z^{2}$. The elliptic orbits are classified by the elements $\{t G, t \in \mathbf{R}-\{0\}\}$, so the equation describing this orbit is

$$
x^{2}+y^{2}-z^{2}=-t^{2} .
$$

Notice that $t$ and $-t$ define the same equation (the same value for the Casimir), but they define different orbits. In fact, the solution of the equation above is a double sheeted hyperboloid, each of the sheets being a different orbit (inside the past and future cone respectively).

Consider now the following automorphism of so(2,1) (in the ordered basis we gave before)

$$
A=\left(\begin{array}{ccc}
-1 & 0 & 0 \\
0 & -1 & 0 \\
0 & 0 & 1
\end{array}\right)
$$


$A$ can in fact be written as $A=\operatorname{Ad}(g)$ with $g$ an element in the complexification of $\mathrm{SO}(2,1)$. In fact,

$$
g=\left(\begin{array}{ccc}
-1 & 0 & 0 \\
0 & 1 & 0 \\
0 & 0 & -1
\end{array}\right),
$$

belongs to $\mathrm{SO}(3)$, the compact real form. Acting on the CSA, $\operatorname{span}\{G\}$, it gives the only Weyl reflection (the Weyl group of $\mathrm{SO}(3)$ is $\{\mathrm{Id},-\mathrm{Id}\}$ ), so $g$ is a representative of the non-trivial element in the Weyl group of $\mathrm{SO}(3)$.

Notice that the CSA of the maximal compact subgroup $\mathrm{SO}(2)$ and of $\mathrm{SO}(3)$ have the same dimension, but the automorphism $A$ is just the Weyl reflection of $\mathrm{SO}(3)$ that is "missing" in $\mathrm{SO}(2)$. $A$ takes a point in one sheet of the hyperboloid and sends it to the other sheet, so $A$ is a diffeomorphism between the two real orbits.

Consider now the subalgebra of polynomials on $\mathcal{G}$ that are invariant under $A$ (since $A^{2}=\mathrm{Id},\{\mathrm{Id}, A\}$ is a subgroup of automorphisms of $\mathrm{so}(2,1)$ ). It is easy to see that it is also a Poisson subalgebra. Moreover, since the Casimir polynomial is invariant under $A$, it is also possible to define a subalgebra of the polynomial algebra of the complex orbit. It is defined over $\mathbf{R}$, since $A$ leaves the real form so $(2,1)$ invariant. This algebra is contained as subalgebra in the algebra of polynomial functions over the real orbit (by polynomial functions we mean polynomials in the ambient space restricted to the orbit).

The implementation of such kind of procedure for more general cases is still under study and will be written elsewhere.

Hyperbolic orbits are classified by the Weyl chamber of the restricted root system. One can take $\mathcal{H}_{\mathcal{P}_{0}}=\operatorname{span}\{\tilde{E}\}$, then $\mathcal{H}_{0}=\operatorname{span}\{\tilde{E}\}$ so the only root is the restricted root. The Weyl chamber is $\left\{t \tilde{E}, t \in \mathbf{R}^{+}\right\}$, so the hyperbolic orbits are given by

$$
x^{2}+y^{2}-z^{2}=t^{2} .
$$

This is a single sheeted hyperboloid, so in this case the orbit is an algebraic manifold.

Finally we have the orbits in the light cone (nilpotent orbits) satisfying

$$
x^{2}+y^{2}-z^{2}=0 .
$$

There are three of them, one for $\mathrm{z}=0$, others for $z>0$ and $z<0$, but we are not studying nilpotent orbits here.

\section{Deformation of the polynomial algebra of regular coadjoint orbits of semisimple groups.}

Definition 3.1. Given a real Poisson algebra $\mathbf{P}$, a formal deformation of $\mathbf{P}$ is an associative algebra $\mathbf{P}_{h}$ over $\mathbf{R}[h]$, where $h$ is a formal parameter, with the following properties: 
a. $\mathbf{P}_{h}$ is isomorphic to $\mathbf{P}[[h]]$ as a $\mathbf{R}[[h]]$-module.

b. The multiplication $*_{h}$ in $\mathbf{P}_{h}$ reduces $\bmod (h)$ to the one in $\mathbf{P}$.

c. $\tilde{F} *_{h} \tilde{G}-\tilde{G} *_{h} \tilde{F}=h\{F, G\} \bmod \left(h^{2}\right)$, where $\tilde{F}, \tilde{G} \in \mathbf{P}_{h}$ reduce to $F, G \in \mathbf{P} \bmod (h)$ and $\{$,$\} is the Poisson bracket in \mathbf{P}$.

If $X$ is a Poisson manifold and $\mathbf{P}=C^{\infty}(X)$ we call $\mathbf{P}_{h}$ a formal deformation of $X$. Some authors also use the term deformation quantization of $X$.

We can also speak of the formal deformation of the complexification $\mathbf{A}$ of a real Poisson algebra. The formal deformation of $\mathbf{A}$ will be an associative algebra $\mathbf{A}_{h}$ with the same properties (a), (b) and (c) where $\mathbf{R}$ has been replaced by $\mathbf{C}$. We want to note here that this doesn't convert the complexification of the symplectic manifold $X$ in a real Poisson manifold of twice the dimension.

We are going to describe first the formal deformation of the polynomial algebra on the complex orbit.

In the first place we will consider $\mathbf{C}[h]$-modules, that is, we will restrict the modules appearing on Definition 3.1 to be modules over $\mathbf{C}[h]$, the algebra of the polynomials in the indeterminate $h$. This will give us immediately the formal deformation by extending to $\mathbf{C}[[h]]$. Notice that our formal deformation will contain a subalgebra that can be specialized to any value of $h \in \mathbf{R}$.

Let $G$ be a complex semisimple Lie group of dimension $n, \mathcal{G}$ its Lie algebra and $U$ the enveloping algebra of $\mathcal{G}$. Let's denote by $T_{A}(V)$ the full tensor algebra of a complex vector space $V$ over a $\mathbf{C}$-algebra $A$. Consider the proper two sided ideal in $T_{\mathbf{C}[h]}(\mathcal{G})$

$$
\mathcal{L}_{h}=\sum_{X, Y \in \mathcal{G}} T_{\mathbf{C}[h]}(\mathcal{G}) \otimes(X \otimes Y-Y \otimes X-h[X, Y]) \otimes T_{\mathbf{C}[h]}(\mathcal{G}) .
$$

We define $U_{h}={ }_{\text {def }} T_{\mathbf{C}[h]}(\mathcal{G}) / \mathcal{L}_{h} . U_{h}$ can be interpreted in the following way:

Let $\mathcal{G}_{h}$ be the Lie algebra over $\mathbf{C}[h] \mathcal{G}_{h}=\mathbf{C}[h] \otimes_{\mathbf{C}} \mathcal{G}$ with Lie bracket

$$
[p(h) X, q(h) Y]_{h}=p(h) q(h)[X, Y]
$$

where [, ] and $[,]_{h}$ denote the brackets in $\mathcal{G}$ and $\mathcal{G}_{h}$ respectively. Then, $U_{h}$ is the universal enveloping algebra of the algebra $\mathcal{G}_{h}$.

We will denote with capital letters elements of the tensor algebras and of $U_{h}$, while we will use lower case letters for the elements of the polynomial algebra over $\mathcal{G}^{*}, \mathbf{C}\left[\mathcal{G}^{*}\right]$. The product of two elements $A, B \in U_{h}$ will be written $A B$. 
Proposition 3.2 (Poincaré-Birkhoff-Witt theorem for $U_{h}$ ). Let $\left\{X_{1}, \ldots\right.$, $\left.X_{n}\right\}$ be a basis for $\mathcal{G}$. Then

$$
1, X_{i_{1}} \cdots X_{i_{k}} \quad 1 \leq i_{1} \leq \cdots \leq i_{k} \leq n
$$

form a basis for $U_{h}$ as $\mathbf{C}[h]$-module.

$U_{h}$ is a free $\mathbf{C}[h]$-module. In particular, $U_{h}$ is torsion free.

Definition 3.3. Let $S(\mathcal{G})=T_{\mathbf{C}}(\mathcal{G}) / \mathcal{L}$, with

$$
\mathcal{L}=\sum_{X, Y \in \mathcal{G}} T_{\mathbf{C}}(\mathcal{G}) \otimes(X \otimes Y-Y \otimes X) \otimes T_{\mathbf{C}}(\mathcal{G}),
$$

be the symmetric algebra of $\mathcal{G}$. The natural homomorphism from $T_{\mathbf{C}}(\mathcal{G})$ to $S(\mathcal{G})$ is an isomorphism if restricted to the symmetric tensors. Let $\lambda$ be the inverse of such isomorphism.

The canonical isomorphism $\mathcal{G}^{* *} \cong \mathcal{G}$, can be extended to an algebra isomorphism $\mathbf{C}\left[\mathcal{G}^{*}\right] \cong S[\mathcal{G}]$ where $\mathbf{C}\left[\mathcal{G}^{*}\right]$ denotes the polynomial algebra over $\mathcal{G}^{*}$. The composition of such isomorphism with $\lambda$ will be called the symmetrizer map.

Let $\left\{X_{1}, \ldots, X_{n}\right\}$ be a basis for $\mathcal{G}$ and $\left\{x_{1}, \ldots, x_{n}\right\}$ the corresponding basis for $\mathcal{G}^{* *} \subset \mathbf{C}\left[\mathcal{G}^{*}\right]$. Then the symmetrizer map Sym : $\mathbf{C}\left[\mathcal{G}^{*}\right] \longrightarrow T_{\mathbf{C}}(\mathcal{G})$ is given by

$$
\operatorname{Sym}\left(x_{1} \cdots x_{n}\right)=\frac{1}{p !} \sum_{s \in S_{p}} X_{s(1)} \otimes \cdots \otimes X_{s(p)}
$$

where $S_{p}$ is the group of permutations of order $p$.

Let $I \subset \mathbf{C}\left[\mathcal{G}^{*}\right]$ be the set of polynomials on $\mathcal{G}^{*}$ invariant under the coadjoint action,

$$
I=\left\{p \in \mathbf{C}\left[\mathcal{G}^{*}\right] \mid p\left(\operatorname{Ad}^{*}(g) \xi\right)=p(\xi) \quad \forall \xi \in \mathcal{G}^{*}, g \in G\right\} .
$$

By Chevalley theorem we have that $I=\mathbf{C}\left[p_{1}, \ldots, p_{m}\right]$, where $p_{1}, \ldots, p_{m}$ are algebraically independent homogeneous polynomials and $m$ is the rank of $\mathcal{G}$.

Definition 3.4. We define a Casimir element in $T_{\mathbf{C}}(\mathcal{G})$ as the image of an invariant polynomial under the symmetrizer map. Since $T(\mathcal{G}) \subset T_{\mathbf{C}[h]}(\mathcal{G})$ Casimirs are also elements of $T_{\mathbf{C}[h]}(\mathcal{G})$. We call Casimir element in $U$ (respectively $U_{h}$ ) an element which is the image of a Casimir element in $T(\mathcal{G})$ (respectively in $T_{\mathbf{C}[h]}(\mathcal{G})$ ) under the natural projection.

It is well known that the Casimir elements lie in the center of $U$. We want now to prove that they also lie in the center of $U_{h}$.

Let's denote by $\tilde{U}_{h_{0}}$ the algebra $U_{h} /\left(\left(h-h_{0}\right) \mathbf{1}\right)$, where $h_{0} \in \mathbf{C}$, and by $e v_{h_{0}}$ the natural projection $U_{h} \longrightarrow \tilde{U}_{h_{0}}$.

Lemma 3.5. Let $P$ be a Casimir in $U_{h}$. Then $e_{h_{0}}(P)$ is in the center of $\tilde{U}_{h_{0}}$. 
Proof. This is because $\tilde{U}_{h_{0}}$ is the universal enveloping algebra of $\mathcal{G}_{h_{0}}$, where $\mathcal{G}_{h_{0}}$ is the complex Lie algebra coinciding with $\mathcal{G}$ as vector space and with bracket $[X, Y]_{h_{0}}=h_{0}[X, Y]$ where $[$,$] is the bracket in \mathcal{G}$.

Theorem 3.6. The Casimir elements lie in the center of $U_{h}$.

Proof. Let $P$ be a Casimir element and let $X_{1}, \ldots, X_{n}$ be generators for $\mathcal{G}$ hence for $\mathcal{G}_{h}$. We need to show: $P X_{i}=X_{i} P$ for all $1 \leq i \leq n$.

$$
P X_{i}-X_{i} P=\sum_{1 \leq i_{1} \leq \cdots \leq i_{k} \leq n} u_{i_{1} \ldots i_{k}}(h) X_{i_{1}} \cdots X_{i_{k}} .
$$

Let us apply the $e v_{h_{0}}$ map.

$$
\begin{aligned}
& e v_{h_{0}}\left(P X_{i}-X_{i} P-\sum_{1 \leq i_{1} \leq \cdots \leq i_{k} \leq n} u_{i_{1} \ldots i_{k}}(h) X_{i_{1}} \cdots X_{i_{k}}\right) \\
& =-\sum_{1 \leq i_{1} \leq \cdots \leq i_{k} \leq n} u_{i_{1} \ldots i_{k}}\left(h_{0}\right) X_{i_{1}} \cdots X_{i_{k}}=0
\end{aligned}
$$

because by Lemma $3.5 e v_{0}\left(P X_{i}-X_{i} P\right)=0$. Since there are no relations among the standard monomials $X_{i_{1}} \cdots X_{i_{k}}$ (Proposition 3.2) we have that $u_{i_{1} \ldots i_{k}}\left(h_{0}\right)=0$. Since this is true for infinitely many $h_{0}$ and since $u_{i_{1} \ldots i_{k}}(h)$ is a polynomial we have that $u_{i_{1} \ldots i_{k}}(h) \equiv 0$.

We now restrict our attention to the regular coadjoint orbits, that is the orbits of regular elements. We recall here the definition of a regular element in $\mathcal{G}^{*}$. Consider the characteristic polynomial of $\operatorname{ad}^{*}(\xi), \xi \in \mathcal{G}^{*}$,

$$
\operatorname{det}\left(T \cdot \mathbf{1}-\operatorname{ad}^{*}(\xi)\right)=\sum_{i \geq m} q_{i}(\xi) T^{i}
$$

where $m=\operatorname{rank} \mathcal{G}^{*}$. The $q_{i}$ 's are invariant polynomials. An element $\xi \in \mathcal{G}^{*}$ is regular if $q_{m}(\xi) \neq 0$. The regular elements are dense in $\mathcal{G}^{*}$ and they are semisimple. In particular the regular elements in a Cartan subalgebra form the interior of the Weyl chambers.

The orbits of regular elements are orbits of maximal dimension $n-m$. Observe also that the 0-eigenspace coincides with the centralizer of $\xi, Z_{\xi}$. A semisimple element $\xi$ is regular if and only if $\operatorname{dim}\left(Z_{\xi}\right)=m$.

Let us fix the coadjoint orbit $C_{\xi}$ of a regular element $\xi \in \mathcal{G}^{*}$. The ideal of polynomials vanishing on $C_{\xi}$ is given by

$$
I_{0}=\left(p_{i}-c_{i 0}, i=1, \ldots, m\right), \quad c_{i 0} \in \mathbf{C},
$$

where the $p_{i}$ have been defined above (see after Definition 3.3). $I_{0}$ is a prime ideal or equivalently the orbit $C_{\xi}$ is an irreducible algebraic variety. (In fact, the orbit of any semisimple element, regular or not, is an irreducible algebraic variety $[\mathbf{K s}])$. 
Let's consider the Casimirs $\hat{P}_{i}=\operatorname{Sym}\left(p_{i}\right)$, where the $p_{1}, \ldots, p_{m}$ are generators for $I$ that satisfy Chevalley theorem. Let $P_{i}$ be the image of $\hat{P}_{i}$ in $U_{h}$. Define the two sided ideal generated by the relations $P_{i}-c_{i}(h), i=1, \ldots, m$ :

$$
I_{h}=\left(P_{i}-c_{i}(h), i=1, \ldots, m\right) \subset U_{h}
$$

for $c_{i}(h)=\sum_{j} c_{i j} h^{j}, c_{i j} \in \mathbf{C}\left(c_{i}(0)=c_{i 0}\right.$, the constants appearing in the definition of $I_{0}$ ).

It is our goal to give a basis of the algebra $U_{h} / I_{h}$ as $\mathbf{C}[h]$-module. We need first a couple of lemmas.

Lemma 3.7. Let $\xi \in \mathcal{G}^{*}$ be a regular element of $\mathcal{G}^{*}$ (or equivalently a point in which the centralizer has dimension equal to the rank of $\left.\mathcal{G}^{*}\right)$. Then $\left(d p_{1}\right)_{\xi}$, $\ldots,\left(d p_{m}\right)_{\xi}$ are linearly independent.

Proof. See $[\mathbf{V a} 3]$.

Lemma 3.8. Let $r$ be a fixed positive integer and let all the notation be as above. Let

$$
\sum_{1 \leq i_{1} \leq \cdots \leq i_{r} \leq m} a_{i_{1} \ldots i_{r}}\left(p_{i_{1}}-k_{i_{1}}\right) \ldots\left(p_{i_{r}}-k_{i_{r}}\right)=0
$$

with $a_{i_{1} \ldots i_{r}} \in \mathbf{C}\left[\mathcal{G}^{*}\right], k_{i_{1}} \ldots k_{i_{r}} \in \mathbf{C}$. Then $a_{i_{1} \ldots i_{r}} \in\left(p_{1}-k_{1}, \ldots, p_{m}-k_{m}\right) \subset$ $\mathbf{C}\left[\mathcal{G}^{*}\right]$.

Proof. By Lemma 3.7 we can choose local coordinates $\left(z_{1}, \ldots, z_{n}\right)$ in a neighborhood of $\xi$ so that $z_{i}=p_{i}-k_{i}, i=1, \ldots, m$. Since $a_{i_{1} \ldots i_{r}}\left(z_{1}, \ldots, z_{n}\right)$ are analytic functions, we can represent them as power series in $z_{1}, \ldots, z_{n}$ :

$$
a_{i_{1} \ldots i_{r}}\left(z_{1}, \ldots, z_{n}\right)=\sum_{\substack{1 \leq j_{1} \leq \ldots j_{s} \leq n \\ 0 \leq s}} a_{i_{1} \ldots i_{r}, j_{1} \ldots j_{s}} z_{j_{1}} \cdots z_{j_{s}} .
$$

This can be rewritten as:

$$
\begin{aligned}
a_{i_{1} \ldots i_{r}}\left(z_{1}, \ldots, z_{n}\right)= & \sum_{\substack{m+1 \leq j_{1} \leq \ldots j_{s} \leq n \\
0 \leq s}} a_{i_{1} \ldots i_{r}, j_{1} \ldots j_{s}} z_{j_{1}} \cdots z_{j_{s}} \\
& +\sum_{\substack{1 \leq l_{1} \leq \cdots l_{t} \leq n \\
l_{1}<m, 1 \leq t}} a_{i_{1} \ldots i_{r}, l_{1} \ldots l_{t}} z_{l_{1}} \cdots z_{l_{t}} .
\end{aligned}
$$

By substituting into the given equation we get:

$$
\begin{aligned}
& \sum_{1 \leq i_{1} \leq \cdots i_{r} \leq m} \sum_{\substack{m+1 \leq j_{1} \leq \cdots j_{s} \leq n \\
0 \leq s}} a_{i_{1} \ldots i_{r}, j_{1} \ldots j_{s}} z_{j_{1}} \cdots z_{j_{s}} z_{i_{1}} \cdots z_{i_{r}} \\
& +\sum_{1 \leq i_{1} \leq \cdots i_{r} \leq m} \sum_{\substack{1 \leq l_{1} \leq \cdots l_{t} \leq n \\
l_{1}<m, 1 \leq t}} a_{i_{1} \ldots i_{r}, l_{1} \ldots l_{t}} z_{l_{1}} \cdots z_{l_{t}} z_{i_{1}} \cdots z_{i_{r}}=0 .
\end{aligned}
$$


Notice that, by the way the sums are defined, and being $r$ fixed, both terms in the above equations have no monomials in common. This implies that

$$
\sum_{1 \leq i_{1} \leq \cdots i_{r} \leq m} \sum_{m+1 \leq j_{1} \ldots j_{s} \leq n} a_{i_{1} \ldots i_{r}, j_{1} \ldots j_{s}} z_{j_{1}} \ldots z_{j_{s}} z_{i_{1}} \ldots z_{i_{r}}=0
$$

from which

$$
a_{i_{1} \ldots i_{r}, j_{1} \ldots j_{s}}=0 \quad \forall 1 \leq i_{1} \ldots i_{r} \leq m, \quad m+1 \leq j_{1} \cdots j_{s} .
$$

This implies

$$
a_{i_{1} \ldots i_{r}}\left(z_{1} \ldots z_{m}\right) \in\left(z_{1} \ldots z_{r}\right) .
$$

That is, locally

$$
a_{i_{1} \ldots i_{r}}=\sum b_{i_{1} \ldots i_{r} j}\left(p_{j}-k_{j}\right) .
$$

So we have obtained that for all $\eta$ in a neighbourhood of $\xi$ :

$$
a_{i_{1} \ldots i_{r}}(\eta)-\sum b_{i_{1} \ldots i_{r}}^{j}(\eta)\left(p_{j}(\eta)-k_{j}\right)=0 .
$$

But since this function is algebraic and $C_{\xi}$ is irreducible this means that this function is identically 0 on $C_{\xi}$. Hence the Lemma is proven.

Let's consider the projection $\pi: U_{h} \longrightarrow U_{h} /(h \mathbf{1}) \cong S(\mathcal{G}) \cong \mathbf{C}\left[\mathcal{G}^{*}\right]$. We have that $\pi(A)=\pi(B)$ if and only if $A \equiv B \bmod h$. To simplify the notation we will denote the element of $\mathbf{C}\left[\mathcal{G}^{*}\right]$ corresponding to $\pi(A)$ by a (same letter, but lower case), as we did for the Casimirs $P_{i}$ before.

Lemma 3.9. Let $k$ be a fixed integer and let

$$
\sum_{i_{1} \leq \cdots i_{k} \leq m} A_{i_{1} \ldots i_{k}}\left(P_{i_{1}}-c_{i_{1}}(h)\right) \cdots\left(P_{i_{k}}-c_{i_{k}}(h)\right) \equiv 0 \quad \bmod h
$$

where $A_{i_{1} \ldots i_{k}} \in U_{h}$ and the $P_{i}$ 's and $c_{i}(h)$ 's have been defined above. Then

$$
\begin{aligned}
& \sum_{i_{1} \leq \cdots i_{k} \leq m} A_{i_{1} \ldots i_{k}}\left(P_{i_{1}}-c_{i_{1}}(h)\right) \cdots\left(P_{i_{k}}-c_{i_{k}}(h)\right) \\
= & h \sum_{i_{1} \leq \cdots i_{k} \leq m} B_{j_{1} \ldots j_{l}, i_{1} \ldots i_{k}}\left(P_{j_{1}}-c_{j_{1}}(h)\right) \cdots \\
& \left(P_{j_{l}}-c_{j_{l}}(h)\right)\left(P_{i_{1}}-c_{i_{1}}(h)\right) \cdots\left(P_{i_{k}}-c_{i_{k}}(h)\right) .
\end{aligned}
$$

Proof. By induction on $N=\max _{i_{1} \ldots i_{k}} \operatorname{deg} a_{i_{1} \ldots i_{k}}$, where, using the the convention above, $a_{i_{1} \ldots i_{k}}=\pi\left(A_{i_{1} \ldots i_{k}}\right)$. Let $N=0$. We have:

$$
\sum a_{i_{1} \ldots i_{k}}\left(p_{i_{1}}-c_{i_{1} 0}\right) \cdots\left(p_{i_{k}}-c_{i_{k} 0}\right)=0
$$

with $a_{i_{1} \ldots i_{k}} \in \mathbf{C}$. By Lemma (3.8) $a_{i_{1} \ldots i_{k}} \in I_{0}$ hence $a_{i_{1} \ldots i_{k}}=0$. This implies that $A_{i_{1} \ldots i_{k}}=h B_{i_{1} \ldots i_{k}}$.

Let's now consider a generic $N$,

$$
\sum a_{i_{1} \ldots i_{k}}\left(p_{i_{1}}-c_{i_{1} 0}\right) \cdots\left(p_{i_{k}}-c_{i_{k} 0}\right)=0 .
$$


By Lemma (3.8)

$$
a_{i_{1} \ldots i_{k}}=\sum_{j} a_{i_{1} \ldots i_{k} j}\left(p_{j}-c_{j 0}\right)
$$

with $\max _{i_{1} \ldots i_{k}} \operatorname{deg} a_{i_{1} \ldots i_{k} j}<N$. Again we have that

$$
A_{i_{1} \ldots i_{k}}=\sum_{j} A_{i_{1} \ldots i_{k} j}\left(P_{j}-c_{j}(h)\right)+h C_{i_{1} \ldots i_{k}} .
$$

Let's substitute $A_{i_{1} \ldots i_{k}}$

$$
\sum A_{i_{1} \ldots i_{k} j}\left(P_{j}-c_{j}(h)\right)\left(P_{i_{1}}-c_{i_{1}}(h)\right) \cdots\left(P_{i_{k}}-c_{i_{k}}(h)\right) \equiv 0 \quad \bmod h .
$$

By induction we have our result.

Lemma 3.10. If $h F \in I_{h}$ then $F \in I_{h}$.

Proof. Since $h F \in I_{h}$ and since the $P_{i}$ are central elements:

$$
h F=\sum A_{i}\left(P_{i}-c_{i}(h)\right) .
$$

We have $\sum A_{i}\left(P_{i}-c_{i}(h)\right) \equiv 0 \bmod h$. Hence, by Lemma 3.9 and also by the fact that $U_{h}$ is torsion free we have our result.

We have shown that $U_{h} / I_{h}$ is a $\mathbf{C}[h]$-module without torsion. We are ready now to show that it is a free module by explicitly constructing a basis. Let's fix a basis $\left\{X_{1}, \ldots, X_{n}\right\}$ of $\mathcal{G}$ and let $x_{1}, \ldots, x_{n}$ be the corresponding elements in $\mathbf{C}\left[\mathcal{G}^{*}\right]$. With this choice $\mathbf{C}\left[\mathcal{G}^{*}\right] \cong \mathbf{C}\left[x_{1}, \ldots, x_{n}\right]$. Let $\left\{x_{i_{1}}, \ldots, x_{i_{k}}\right\}_{\left(i_{1}, \ldots, i_{k}\right) \in \mathbf{A}}$ be a basis in of $\mathbf{C}\left[\mathcal{G}^{*}\right] / I_{0}$ as $\mathbf{C}$-module, where $\mathbf{A}$ is a set of multiindices appropriate to describe the basis. In particular, we can take them such that $i_{1} \leq \cdots \leq i_{k}$.

Proposition 3.11. The monomials $\left\{X_{i_{1}} \cdots X_{i_{k}}\right\}_{\left(i_{1}, \ldots, i_{k}\right) \in \mathbf{A}}$ are linearly independent in $U_{h} / I_{h}$.

Proof. Suppose that there exists a linear relation among the $X_{i_{1}}, \cdots X_{i_{k}}$ 's, $\left(i_{1}, \ldots, i_{k}\right) \in \mathbf{A}$ and let $G \in I_{h}$ be such relation,

$$
G=G_{0}+G_{1} h+\cdots, \quad G_{i} \in \operatorname{span}_{\mathbf{C}}\left\{X_{i_{1}} \cdots X_{i_{k}}\right\}_{\left(i_{1} \ldots i_{k}\right) \in \mathbf{A}} .
$$

Assume $G_{i}=0, i<k, G_{k} \neq 0$. We can write $G=h^{k} F$, with

$$
F=F_{0}+F_{1} h+\cdots, \quad F_{0} \neq 0 .
$$

Since $h^{k} F \in I_{h}$ by hypothesis, using Lemma (3.10) we have that $F \in I_{h}$, that is

$$
F=\sum A_{i}\left(P_{i}-c_{i}(h)\right),
$$

and reducing $\bmod h$,

$$
f=\sum a_{i}\left(p_{i}-c_{i 0}\right)
$$


This would mean that $f$ represents a non-trivial relation among the monomials $\left\{x_{i_{1}} \cdots x_{i_{k}}\right\}_{\left(i_{1} \ldots i_{k}\right) \in \mathbf{A}}$ in $C\left[\mathcal{G}^{*}\right] / I_{0}$, which is a contradiction, so the linear independence is proven.

We want to give a procedure to construct a basis on $\mathbf{C}\left[\mathcal{G}^{*}\right] / I_{0}$ starting from a set of generators of $\mathbf{C}\left[\mathcal{G}^{*}\right], S=\left\{x_{i_{1}} \cdots x_{i_{k}}\right\} \forall 1 \leq i_{1} \leq \cdots i_{k} \leq n$. As a linear space $I_{0}=\operatorname{span}_{\mathbf{C}}\left\{x_{i_{1}} \cdots x_{i_{k}}\left(p_{i}-c_{i}\right)\right\}$. Every element of the set that spans $I_{0}$ will provide one relation that will allow us to eliminate at most one element of the set $S$. We can choose to eliminate successively the greatest element with respect to lexicographic ordering. This means that any monomial in $S$ will be expressed in terms of monomials of degree less or equal to its degree.

Remarks 3.12. We want to make two remarks that will be used later.

1. An arbitrary monomial $x_{j_{1}} \cdots x_{j_{r}}$ in $\mathbf{C}\left[\mathcal{G}^{*}\right]$ can be written as:

$$
x_{j_{1}} \cdots x_{j_{r}}=\sum_{\substack{k \leq r \\\left(m_{1}, \ldots, m_{k}\right) \in \mathbf{A}}} a_{m_{1} \ldots m_{k}}^{j_{1} \ldots j_{r}} x_{m_{1}} \cdots x_{m_{k}}+\sum_{i, d_{i}+g_{i} \leq r} b_{i}\left(p_{i}-c_{i}\right)
$$

where $b_{i}$ is polynomial of degree $g_{i}, d_{i}=\operatorname{deg} p_{i}$ and $a_{m_{1} \ldots m_{k}}^{j_{1} \ldots j_{r}} \in \mathbf{C}$.

2. Let $A \in U_{h}, A \neq 0, A \in \operatorname{span}_{\mathbf{C}}\left\{X_{j_{1}} \cdots X_{j_{p}}\right\}_{p \leq r}, j_{1} \cdots j_{p}$ not necessarily ordered. If $A \equiv 0 \bmod h$, then $A=h B, B \in \operatorname{span}_{\mathbf{C}}\left\{X_{i_{1}} \cdots X_{i_{p}}\right\} \underset{i_{1} \leq \cdots \leq i_{p}}{p<r}$.

Next proposition will show the generation, so we will have a basis.

Proposition 3.13. The standard monomials $\left\{X_{i_{1}} \cdots X_{i_{k}}\right\}$ with $\left(i_{1}, \ldots, i_{k}\right)$ $\in \mathbf{A}$ generate $U_{h} / I_{h}$ as $\mathbf{C}[h]$-module.

Proof. By Proposition 3.2 ( $\mathrm{PBW}$ theorem in $U_{h}$ ) it is sufficient to prove that

$$
X_{j_{1}} \cdots X_{j_{r}} \in \operatorname{span}_{\mathbf{C}[h]}\left\{X_{i_{1}} \cdots X_{i_{k}}\right\}_{\left(i_{1}, \ldots, i_{k}\right) \in \mathbf{A}}
$$

where $1 \leq j_{1} \leq \cdots j_{r} \leq n$ and $X_{j_{1}} \cdots X_{j_{r}}$ denotes also the projection onto $U_{h} / I_{h}$ of the standard monomial.

We proceed by induction on $r$. For $r=0$ it is clear. For generic $r$ we write (see Remark 3.12)

$$
x_{j_{1}} \cdots x_{j_{r}}=\sum_{\substack{k \leq r \\\left(m_{1}, \ldots, m_{k}\right) \in \mathbf{A}}} a_{m_{1} \ldots m_{k}}^{j_{1} \ldots j_{r}} x_{m_{1}} \cdots x_{m_{k}}+\sum_{i, d_{i}+g_{i} \leq r} b_{i}\left(p_{i}-c_{i}\right) .
$$

Lifting this equation from the symmetric algebra to the enveloping algebra we have

$$
X_{j_{1}} \cdots X_{j_{r}}-\sum_{\substack{k \leq r \\\left(m_{1}, \ldots, m_{k}\right) \in \mathbf{A}}} a_{m_{1} \ldots m_{k}}^{j_{1} \ldots j_{r}} X_{m_{1}} \cdots X_{m_{k}}-\sum_{i} B_{i}\left(P_{i}-c_{i}(h)\right)=h B
$$

where, by the Remark 2 in $3.12, B \in \operatorname{span}\left\{X_{i_{1}} \cdots X_{i_{p}}\right\}_{p<r}$. Applying the induction hypothesis, we have our result. 
Let $\mathbf{C}_{h}\left[\mathcal{G}^{*}\right]=\mathbf{C}[h] \otimes \mathbf{C}\left[\mathcal{G}^{*}\right], I_{0}^{\prime}=\mathbf{C}[h] \otimes I_{0}$. We are now ready to prove the following theorem:

Theorem 3.14. Let the notation be as above. We have that $U_{h} / I_{h}$ has the following properties:

1. $U_{h} / I_{h}$ is isomorphic to $\mathbf{C}_{h}\left[\mathcal{G}^{*}\right] / I_{0}^{\prime}$ as a $\mathbf{C}[h]$-module.

2. The multiplication in $U_{h} / I_{h}$ reduces $\bmod (h)$ to the one in $\mathbf{C}\left[\mathcal{G}^{*}\right] / I_{0}^{\prime}$.

3. If $F G-G F=h P, F, G, P \in U_{h} / I_{h}$, then $p=\{f, g\}$, where $\{$,$\} is the$ Poisson bracket on the orbit defined by $I_{0}$. (We are using the same convention, $f=\pi(F)$.)

Proof.

1. It is a consequence of Propositions 3.11 and 3.13.

2. It is trivial.

3. This property is satisfied by the multiplication in $U_{h}$ and the Poisson bracket in $\mathbf{C}\left[\mathcal{G}^{*}\right]$ (see $[\mathbf{K o}],[\mathbf{C P}],[\mathbf{K i}]$ ). The Poisson bracket in the $\mathbf{C}\left[\mathcal{G}^{*}\right] / I_{0}$ is induced from the one in $\mathbf{C}\left[\mathcal{G}^{*}\right]$, it is enough to see that $p$ will not depend on the representative chosen in $U_{h} / I_{h}$, which is trivial.

It is now immediate to obtain the properties of Definition 3.1 when we consider the extension of $\mathbf{C}[h]$ to $\mathbf{C}[[h]]$. We define

$$
\begin{gathered}
\mathbf{C}_{[h]}\left[\mathcal{G}^{*}\right]=\mathbf{C}[[h]]\left[\mathcal{G}^{*}\right] \quad I_{[0]} \subset \mathbf{C}_{[h]}\left[\mathcal{G}^{*}\right] \\
U_{[h]}=T_{\mathbf{C}[[h]]}(\mathcal{G}) / \mathcal{L}_{[h]} \quad I_{[h]} \subset U_{[h]}
\end{gathered}
$$

being $I_{[0]}$ and $I_{[h]}$ the ideals obtained by extending $I_{0}$ and $I_{h}$ to $C_{[h]}\left[\mathcal{G}^{*}\right]$ and $U_{[h]}$ respectively.

Theorem 3.15. $U_{[h]} / I_{[h]}$ is a formal deformation (or a deformation quantization) of $\mathbf{C}_{[h]}\left[\mathcal{G}^{*}\right] / I_{[0]}$.

We want to note here that whatever is the real form chosen, the deformed algebra is defined over $\mathbf{R}$, provided $c_{i j} \in \mathbf{R}$. Care should be taken, nevertheless, in choosing the appropriate generators of $I_{0}$ with real coefficients and this is always possible $([\mathbf{B o}])$.

Finally we want to come back to Example 2.1 and exhibit the deformed algebra.

Example 3.16. Let $G=S L_{2}(\mathbf{C})$. The standard basis for $\mathcal{G}=s l_{2}(\mathbf{C})$ is $\{H, X, Y\}$ with commutation relations

$$
[H, X]=2 X \quad[H, Y]=-2 Y \quad[X, Y]=H .
$$

We identify $\mathcal{G}$ and $\mathcal{G}^{*}$ via the Cartan Killing form. The only independent invariant polynomial is:

$$
p=\frac{1}{4} h^{2}+x y
$$


or, in terms of the compact generators

$$
\begin{aligned}
& E=\frac{1}{2}(X-Y) \quad F=i / 2(X+Y) \quad G=i / 2 H \\
& p=-\left(e^{2}+f^{2}+g^{2}\right) .
\end{aligned}
$$

The orbit $C_{\xi}$ of the regular semisimple element $\xi=\left(\begin{array}{cc}i a / 2 & 0 \\ 0 & -i a / 2\end{array}\right)$ (see the fundamental representation in the next section) has coordinate ring $\mathbf{C}[h, x, y] /\left(e^{2}+f^{2}+g^{2}-a^{2}\right)$. So we have that

$$
U_{[[h]]} /\left(E^{2}+F^{2}+G^{2}-a^{2}+c_{1} h+\cdots+c_{l} h^{l}\right)
$$

is a formal deformation of $C_{\xi}$. If one chooses $a, c_{1}, \ldots, c_{l}$ to be real, then it becomes the complexification of a formal deformation of the real orbit $C_{\xi} \cap s u(2)$.

To go to the non-compact form it is enough to take the basis $\{\tilde{E}=i E, \tilde{F}=$ $i F, G\}$. The deformed algebra is

$$
U_{[[h]]} /\left(-\tilde{E}^{2}-\tilde{F}^{2}+G^{2}-a^{2}+c_{1} h+\cdots+c_{l} h^{l}\right) .
$$

A basis for $U / I_{0}$ is

$$
\left\{g^{m} \tilde{e}^{n} \tilde{f}^{\mu}\right\}_{\substack{m, n=0,1,2 \ldots \\ \mu=0,1}}
$$

The subalgebra invariant under the automorphism $A$ of Example 2.1, has instead a basis

$$
\left\{g^{m} \tilde{e}^{2 n-m} \tilde{f}^{\mu}\right\}_{\substack{m, n=0,1,2 \ldots \\ \mu=0,1}} .
$$

We can also express this algebra in terms of the set of commutative generators

$$
v_{1}=g^{2}, \quad v_{2}=\tilde{e}^{2}, \quad v_{3}=g \tilde{e}, \quad v_{4}=\tilde{f}
$$

with relations

$$
v_{3}^{2}=v_{1} v_{2}, \quad v_{1}-v_{2}-v_{4}^{2}=a^{2} .
$$

It is clear that this algebra separates the points of the real orbit. Since the Casimir element is invariant under the automorphism $A$ (extended to $U_{h}$ ), it restricts to an automorphism of $U_{h} / I_{h}$. Analogously to the commutative case, the subalgebra of $U_{h} / I_{h}$ invariant under $A$ can be given in terms of the generators

$$
V_{1}=G^{2}, \quad V_{2}=\tilde{E}^{2}, \quad V_{3}=G \tilde{E}, \quad V_{4}=\tilde{F}
$$

and relations

$$
V_{3}^{2}=V_{1} V_{2}-h V_{3} V_{4}-h^{2} V_{1}, \quad V_{1}-V_{2}-V_{4}^{2}=c(h),
$$


in addition to the commutation relations

$$
\begin{aligned}
& V_{4} V_{1}-V_{1} V_{4}=h\left(2 V_{3}\right)-h^{2} V_{4}, \quad V_{4} V_{2}-V_{2} V_{4}=h\left(2 V_{3}\right)-h^{2} V_{4}, \\
& V_{4} V_{3}-V_{3} V_{4}=h\left(V_{1}+V_{2}\right), \quad V_{3} V_{1}-V_{1} V_{3}=-h\left(2 V_{1} V_{4}\right)-h^{2} V_{3}, \\
& V_{3} V_{2}-V_{2} V_{3}=h\left(V_{4} V_{2}+V_{2} V_{4}\right)+h^{2} V_{3}-h^{3} V_{4}, \\
& V_{2} V_{1}-V_{1} V_{2}=-h\left(2 V_{3} V_{4}\right)+h^{2}\left(V_{4}^{2}-V_{2}-V_{1}\right) .
\end{aligned}
$$

\section{Geometric quantization of $S^{2}$.}

The subject of geometric quantization is a very vast one and we do not intend to make a review here. Many excellent reviews exist in the literature (see for example $[\mathbf{P u}],[\mathbf{V o}]$ ). We will try to explain only what is needed to understand the geometric quantization of our particular case, $S^{2}$. Some of the results we exhibit here date back to [So]. We will follow closely the scheme of $[\mathbf{V o}]$, because there the importance of constructing the algebra of observables is emphasized.

Consider a classical system with phase space $X$ and a group $G$ of symmetries. This means that $G$ is a group of symplectomorphisms of the symplectic manifold $X$,

$$
g \in G, \quad g: X \mapsto X \quad \text { satisfying } \quad g^{*} \omega=\omega,
$$

where $\omega$ is the symplectic form on $X$. The Hamiltonian is a $G$-invariant function, that is, $g H=H$, so $G$ is a group of symmetries of the equations of motion.

We want to find a quantization of the classical system that preserves the symmetry under the group $G$. The goal of geometric quantization is to construct the Hilbert space $\mathcal{H}_{X}$ and the algebra of quantum observables $\mathbf{A}_{h}$ acting on $\mathcal{H}_{X}$ using only the geometrical elements of the classical system. This construction should be "natural", that is, the action of $G$ on $X$ as symplectomorphisms should induce a unitary representation of $G$ on $\mathcal{H}_{X}$ and an action of $G$ on $\mathbf{A}_{h}$. This action should reduce to the conjugation by the unitary representation on the operators on $\mathcal{H}_{X}$ representing the elements of $\mathbf{A}_{h}$.

Integral orbit data.

Let $\xi \in \mathcal{G}_{0}{ }^{*}$ and let $G_{\xi}$ the isotropy group of $\xi$ and $\mathcal{G}_{0 \xi}$ the corresponding Lie algebra. It is clear that for $Z \in \mathcal{G}_{0 \xi}, \operatorname{ad}_{Z}^{*} \xi=0$, which implies

$$
\xi([Z, Y])=0, \quad \forall Y \in \mathcal{G}_{0} .
$$

Suppose that we have a character $\tau$ of $G_{\xi}$ satisfying

$$
\tau\left(\mathrm{e}^{X}\right)=\mathrm{e}^{i \xi(X)}, \quad Z \in \mathcal{G}_{0 \xi} .
$$


Such character is called an integral orbit datum. Notice that property (4.1) is essential. Also, $\xi$ must be such that $\xi(Z)=2 \pi m, m \in \mathbf{Z}$ whenever $e^{Z}=$ Id.

From an integral orbit datum we can construct a unitary representation of $G$ by induction. We consider the induced vector bundle $E\left(G / G_{\xi}, \mathbf{C}_{\tau}\right)=$ $(G \times \mathbf{C}) / \tau$, where the equivalence relation is given by

$$
(g, v) \approx\left(g h^{-1}, \tau(h) v\right), \quad h \in G_{\xi} .
$$

We can describe the sections on this bundle by functions $f: G \mapsto \mathbf{C}$ satisfying

$$
f(g h)=\tau(h)^{-1} f(g) .
$$

By considering the compactly supported sections, and from the fact that there is a a $G$-invariant measure on $G / G_{\xi}$ the construction of the Hilbert space is straightforward, with bilinear form

$$
\left\langle f_{1}, f_{2}\right\rangle=\int_{G / G_{\xi}} f_{1} \bar{f}_{2} .
$$

The problem is that this representation is not necessarily irreducible. Nevertheless, in many cases (like for elliptic orbits), it is possible to restrict naturally the space of sections (4.2) to an irreducible component. We are then interested in computing the integral orbit data for $\mathrm{SU}(2)$.

The Lie algebra of $\mathrm{SU}(2)$ is spanned by the matrices

$$
G=\frac{i}{2} \sigma_{3}, \quad E=\frac{i}{2} \sigma_{2}, \quad F=\frac{i}{2} \sigma_{1}
$$

with

$$
\sigma_{1}=\left(\begin{array}{cc}
0 & 1 \\
1 & 0
\end{array}\right), \quad \sigma_{2}=\left(\begin{array}{cc}
0 & -i \\
i & 0
\end{array}\right), \quad \sigma_{3}=\left(\begin{array}{cc}
1 & 0 \\
0 & -1
\end{array}\right),
$$

and commutation relations ${ }^{1}$

$$
[E, F]=G, \quad[F, G]=E, \quad[G, E]=F .
$$

Consider $\xi_{a} \in \mathcal{G}_{0}{ }^{*}$ such that $\xi_{a}(x E+y F+z G)=a z$. The isotropy group is

$$
G_{\xi_{a}}=\left\{\mathrm{e}^{z G}, z \in \mathbf{R}\right\}=\left\{\left(\begin{array}{cc}
\mathrm{e}^{i z / 2} & 0 \\
0 & \mathrm{e}^{-i z / 2}
\end{array}\right), z \in \mathbf{R}\right\}
$$

with Lie algebra $\mathcal{G}_{0 \xi_{a}}=\operatorname{span}\{G\}$. If $z=4 \pi n, n \in \mathbf{Z}$, then $e^{z G}=\mathrm{Id}$, so in order to have an integral orbit datum,

$$
\xi_{a}(4 \pi n G)=4 \pi n a \in 2 \pi \mathbf{Z} \quad \forall n,
$$

which is possible if and only if $a \in \mathbf{Z} / 2$.

${ }^{1}$ The spin operators which are used in physics are given by $G^{\prime}=-i \hbar G, E^{\prime}=$ $-i \hbar E, F^{\prime}=-i \hbar F$. We can reintroduce $\hbar=h / 2 \pi$ in the analysis with this rescaling, the multiplication by $-i$ changing a representation by antihermitian operators of $\mathrm{SU}(2)$ to hermitian operators. 
The Cartan-Killing form allows the identification of $\mathcal{G}_{0}$ and $\mathcal{G}_{0}{ }^{*}$, also intertwining the adjoint and coadjoint representations. It is given by

$$
\langle X, Y\rangle=-\frac{1}{2} \operatorname{Tr}(\operatorname{ad} X \operatorname{ad} Y), \quad X, Y \in \mathcal{G}_{0}
$$

that is,

$$
\langle E, E\rangle=\langle F, F\rangle=\langle G, G\rangle=1
$$

and the rest 0 . So $\xi_{a} \approx a G$, and the orbit is given by the Casimir polynomial

$$
C=x^{2}+y^{2}+z^{2}=a^{2} .
$$

We conclude that only orbits with half integer radius have integral orbit data. We will denote by $\tau_{m}$ the corresponding integral orbit datum, $\tau_{m}\left(e^{z G}\right)=\left(e^{i z / 2}\right)^{m}$.

It is easy to convince oneself that the representation in the space of functions (4.2) is far too large to be irreducible. To overcome this problem we need to further restrict the space of sections. We will do that with the help of a complex polarization.

Complex polarization and Hilbert space.

Elliptic orbits have a $G$-invariant complex structure. We define this complex structure following [Vo]. From now on we use the identification between $\mathcal{G}_{0}$ and $\mathcal{G}_{0}{ }^{*}$ given by the Cartan-Killing form, so we will use alternatively $\xi=\xi_{X} \in \mathcal{G}_{0}{ }^{*}$ with $X \in \mathcal{G}_{0}$.

Theorem 4.1. Let $X \in \mathcal{G}_{0}$ be such that ad $d_{X}$ has only imaginary eigenvalues. Let $\mathcal{G}$ be the complexified Lie algebra of $\mathcal{G}_{0}$ and let $\mathcal{G}^{t}(t \in \mathbf{R})$ be the t-eigenspace of ad $d_{i X}$. Then

$$
\mathcal{G}=\sum_{t \in \mathbf{R}} \mathcal{G}^{t}, \quad\left(\mathcal{G}_{0 X}\right)_{c}=\mathcal{G}_{X}=\mathcal{G}^{0}
$$

is a gradation of $\mathcal{G}$. We define

$$
\mathcal{P}_{X}=\sum_{t \geq 0} \mathcal{G}^{t}, \quad \mathcal{N}_{X}=\sum_{t>0} \mathcal{G}^{t}
$$

The following properties are satisfied

a. $\mathcal{G}^{s}$ and $\mathcal{G}^{t}$ are orthogonal unless $s=-t$.

b. $\overline{\mathcal{G}}^{s}=\mathcal{G}^{-s}$. (Bar means complex conjugation with respect to the real form $\mathcal{G}_{0}$.)

c. The adjoint action of $G_{X}$ preserves $\mathcal{G}^{t}$.

$\mathcal{G} / \mathcal{G}_{X} \approx T_{\xi_{X}}\left(G \cdot \xi_{X}\right)_{c}$ is the complexified tangent space at the identity coset. The $G$-invariant complex structure can be characterized by requiring that $\mathcal{P}_{X} / \mathcal{G}_{X}$ is the antiholomorphic tangent space at the identity coset. 
Let us write down the standard complex structure on $S^{2}$ to relate it with this formalism. Let $V=x E+y F+z G=x \partial_{x}+y \partial_{y}+z \partial_{z} \in \mathcal{G}_{0}$. We take a representative $a G$ for the orbit of radius $a$,

$$
x^{2}+y^{2}+z^{2}=a^{2} .
$$

Stereographic coordinates are given in terms of the embedding coordinates by

$$
\begin{array}{ll}
V_{1}=S^{2}-\{(0,0,-a)\}, & u_{1}=\frac{a x}{z+a}, \quad v_{1}=\frac{a y}{z+a} \\
V_{2}=S^{2}-\{(0,0, a)\}, & u_{2}=\frac{a x}{z-a}, \quad v_{2}=\frac{a y}{z-a} .
\end{array}
$$

The action of $\mathrm{SU}(2)$ is the one induced by the adjoint representation of $\mathrm{SU}(2)$.

Let $x_{1}: U_{1} \longrightarrow \mathbf{C}, x_{2}: U_{2} \longrightarrow \mathbf{C}$ be the projective coordinates for the complex projective space $\mathbf{P}^{1}=U_{1} \cup U_{2}$. If we identify

$$
x_{1} \equiv-v_{1}+i u_{1}, \quad x_{2} \equiv-v_{2}-i u_{2},
$$

we obtain a diffeomorphism $S^{2} \approx \mathbf{P}^{1}$. This gives to $S^{2}$ the complex structure mentioned above. For this particular choice, the action of $\mathrm{SU}(2)$ obtained from the three dimensional representation restricted to $S^{2}$ coincides with the one obtained from the fundamental representation with the projective structure.

We write now the complexification of $\operatorname{su}(2), \operatorname{sl}(2, \mathbf{C})$, in the standard basis

$$
H=-i 2 G, \quad X=E-i F, \quad Y=-E-i F .
$$

The eigenvalues of $i a G$ are $-a, 0,+a$ and the corresponding eigenspaces are

$$
\mathcal{G}^{0}=\operatorname{span}\{G\}, \quad \mathcal{G}^{a}=\operatorname{span}\{Y\}, \quad \mathcal{G}^{-a}=\operatorname{span}\{X\} .
$$

The tangent space at the North pole $(x=y=0, z=a)$ is spanned by $\partial_{x}, \partial_{y} \in \mathcal{G}_{0} / \mathcal{G}_{0 a G}$ and in terms of the stereographic coordinates,

$$
\partial_{x}=\frac{1}{2} \partial_{u_{1}}, \quad \partial_{y}=\frac{1}{2} \partial_{v_{1}} .
$$

In the complexified tangent space,

$$
X=\partial_{x}-i \partial_{y}=\frac{i}{2}\left(-\partial_{v_{1}}-i \partial_{u_{1}}\right), \quad Y=-\partial_{x}-i \partial_{y}=\frac{i}{2}\left(-\partial_{v_{1}}+i \partial_{u_{1}}\right),
$$

and since the complex coordinate is $x_{1}=-v_{1}+i u_{1}$,

$$
\mathcal{G}^{a}=\operatorname{span}\{Y\}=\operatorname{span}\left\{\partial_{\bar{x}_{1}}\right\} .
$$

Definition 4.2. A $G$-invariant complex polarization is a lagrangian subspace of the complexified tangent bundle at $\xi, T_{\xi}(G \cdot \xi)_{c} \approx \mathcal{G} / \mathcal{G}_{\xi}$. 
We remind that a subspace is a lagrangian subspace if the symplectic form is 0 on that subspace and its dimension is half the dimension of the symplectic manifold. Because of property a in Theorem $4.1, \mathcal{P}_{X} / \mathcal{G}_{X}$ is a lagrangian subspace and then a complex polarization.

Consider now an integral orbit datum, $\tau$. One can prove that $d \tau$ extends to a representation $\phi$ of $\mathcal{P}_{X}$. This extension satisfies $\left.\phi\right|_{\mathcal{N}_{X}}=0$. The induced bundle associated to the character $\tau, E\left(G / G_{X}, \mathbf{C}_{\tau}\right)$ has also a complex structure and the holomorphic sections are characterized by

$$
Z . f=-\phi(Z) f \quad Z \in \mathcal{P}_{X}
$$

where $f: G \mapsto \mathbf{C}$ satisfies $f(g h)=\tau(h)^{-1} f(g), g \in G, h \in G_{X}$. We will see that in our case this construction gives directly the Hilbert space. For other groups, further corrections are needed.

It is easy to see that for $\mathrm{SU}(2)$ the principal bundle $E(\mathrm{SU}(2) / \mathrm{U}(1), \mathrm{U}(1))$ is only a reduction of the principal bundle given by the natural projection

$$
\pi: \mathbf{C}^{2}-\{0\} \mapsto S^{2} \approx \mathbf{P}_{1}
$$

that we call $\Theta\left(S^{2}, \mathbf{C}^{*}\right)$. The corresponding associated bundles by the representation $\tau_{m}$ (extended to $\mathbf{C}^{*}$ ), will be denoted by $E(m), \Theta(m) . \Theta(m)$ is an holomorphic vector bundle, whose sections satisfy (4.3), which in this case is simply

$$
\partial_{\bar{x}_{1}} f=0 .
$$

Line bundles over $S^{2}$ are well studied. A holomorphic section on $\Theta(m)$

$$
s: \mathbf{P}_{1} \mapsto \Theta(m) / \quad \pi \circ s=\operatorname{id}_{\mathbf{P}_{1}},
$$

can be given in terms of a function

$$
\tilde{s}: \mathbf{C}^{2}-\{0\} \mapsto \mathbf{C}_{m}
$$

$\left((\lambda, \rho) \in \mathbf{C}^{2}-\{0\}\right)$ satisfying $\tilde{s}(\lambda \cdot \gamma, \lambda \cdot \rho)=\lambda^{m} \tilde{s}(\gamma, \rho)$ where $\tilde{s}$ is a homogeneous polynomial in two variables of degree $m$. The group $\mathrm{SU}(2)$ naturally acts on this space of sections, constituting the $(m+1)$-dimensional (unitary) irreducible representation of $\mathrm{SU}(2)$.

We see that geometric quantization associates quite naturally to the orbit a Hilbert space where the group $G$ acts. The last step now is to find the algebra of quantum observables.

Quantum observables.

Following [Vo], the algebra of observables is the algebra of "twisted differential operators" $[\mathbf{V o}]$ on sections of the bundle given by the polarization (real or complex). These operators are endomorphisms of the space of sections of the bundle satisfying certain conditions (which make plausible the name of "differential operators"). We will not give here the general definition, but we will work with the $\mathrm{SU}(2)$-bundles using the description given above. 
Consider the space of functions $f: \mathbf{C}^{2}-\{0\} \longrightarrow \mathbf{C}$, and $(\gamma, \rho)$ global coordinates on $\mathbf{C}^{2}-\{0\}$. Consider the algebra of differential operators generated by the elements

$$
\gamma \partial_{\gamma}, \quad \gamma \partial_{\rho}, \quad \rho \partial_{\gamma}, \quad \rho \partial_{\rho}
$$

We denote this algebra by $\mathcal{D}$. It is a filtered algebra (each of the elements above has degree 1).

The algebra of twisted differential operators on $\Theta(m)$ is

$$
\mathcal{D}_{m}=\mathcal{D} /(D-m \mathrm{Id})
$$

where $D=\gamma \partial_{\gamma}+\rho \partial_{\rho}$ is an element in the center of $\mathcal{D}$.

We want to give a presentation for $\mathcal{D}_{m}$ and compare it to the algebra $U_{h} / I_{h}$ obtained in Section 3.

Consider now $\mathcal{U}$ the universal enveloping algebra of the Lie algebra $\mathrm{su}(2)^{\mathrm{C}}$ $\approx \operatorname{sl}(2, \mathbf{C})$. Let $\{X, Y, H\}$ be the standard basis of sl(2,C) (Example 3.16).

Lemma 4.3. The filtered algebra homomorphism $p: \mathcal{U} \longrightarrow \mathcal{D}$, given by

$$
p(X)=-\gamma \partial_{\rho}, \quad p(Y)=-\rho \partial_{\gamma}, \quad p(H)=-\gamma \partial_{\gamma}+\rho \partial_{\rho}
$$

is injective.

Proof. Notice that $\mathcal{D}$ acts on the space $P_{m}=$ \{homogeneous polynomials of degree $m\}$. We denote by $R_{m}: \mathcal{D} \longrightarrow \operatorname{End}\left(P_{m}\right)$ this representation. Notice that $\tilde{R}_{m}=R_{m} \circ p$ is the $m+1$-dimensional irreducible representation of $\mathrm{su}(2)$. Since we have that $\tilde{R}_{m}(Z)=0 \quad Z \in \mathcal{U} \forall m \Rightarrow Z=0$ [HC], it follows that $p$ is an injective map.

\section{Lemma 4.4.}

$$
\mathcal{D} \cong \mathcal{U} \otimes \operatorname{span}\{D\} /\left(C-\frac{D}{2}\left(\frac{D}{2}+1\right)\right)
$$

where $C=\frac{1}{2}\left(X Y+Y X+\frac{1}{2} H^{2}\right)$ is the Casimir element in $\mathcal{U}$.

Proof. Define the Lie algebra homomorphism

$$
\mathcal{U} \otimes \operatorname{span}\{D\} \stackrel{S}{\longrightarrow} \mathcal{D}
$$

as $S(W \otimes D)=p(W) D$. Since $\{p(X), p(Y), p(H), D\}$ generate $\mathcal{D}, S$ is surjective. We want to show that $\operatorname{ker} S=I$, where $I=(C-D / 2(D / 2+1))$. One can check directly that $I \subset \operatorname{ker} S$. We prove $\operatorname{ker} S \subset I$ by contradiction.

Observe first that any element $P \in \mathcal{U} \otimes \operatorname{span}\{D\} /\left(C-\frac{D}{2}\left(\frac{D}{2}+1\right)\right)$ can be written as $A D+B$. In fact, let $P=\sum_{k=0}^{N} A_{k} D_{k}$. By induction on $N$. The cases of $N=0,1$ are obvious. Let $N>1$.

$$
P=A_{N} D^{N-2}(4 C-2 D)+\sum_{k=0}^{N-1} A_{k} D_{k} .
$$


By induction we have our result.

Let $P_{N-1}=B_{1} D+B_{0}$ be a non zero element in $\operatorname{ker} S$ that is not in $I$. Let us construct the combination

$$
P_{N-1}^{\prime}=B_{1} P_{1}+\frac{1}{4} P_{N-1}=\left(\frac{1}{4} B_{0}-\frac{1}{2} B_{1}\right) D+B_{1} C
$$

it is clear that $P_{N-1}^{\prime}$ doesn't belong to $I$ unless it is identically 0 , that is, $B_{0}=B_{1}=0$. In this case $P_{N-1}$ is also 0 , against the hypothesis. So $P_{N-1}^{\prime}$ is in ker $S$ and not in $I$. Let us construct now the combination

$$
P_{N}=\left(\frac{1}{4} B_{0}-\frac{1}{2} B_{1}\right) P_{N-1}-P_{N-1}^{\prime} B_{1}=\frac{1}{4} B_{0}^{2}-\frac{1}{2} B_{1} B_{0}-B_{1}^{2} C .
$$

Since $P_{N} \in \operatorname{ker} S$ and $P_{N}$ does not contain $D$, by the injectivity of $p$ we must have $P_{N}=0$, that is

$$
\frac{1}{4} B_{0}^{2}-B_{1}^{2} C=\frac{1}{2} B_{1} B_{0}
$$

Similarly if we construct

$$
P_{N}^{\prime}=P_{N-1}\left(\frac{1}{4} B_{0}-\frac{1}{2} B_{1}\right)-P_{N-1}^{\prime} B_{1}=\frac{1}{4} B_{0}^{2}-\frac{1}{2} B_{0} B_{1}-B_{1}^{2} C .
$$

$P_{N}^{\prime}$ must also be 0 , so we have that

$$
\frac{1}{4} B_{0}^{2}-B_{1}^{2} C=\frac{1}{2} B_{0} B_{1}
$$

It follows that $B_{1}$ and $B_{0}$ commute. Lets us rewrite any of these two relations as

$$
\left(B_{0}-B_{1}\right)^{2}=(4 C+1) B_{1}^{2} .
$$

We show that this relation cannot be satisfied unless $B_{0}=B_{1}=0$ and this will be a contradiction. Consider the homomorphism from the (filtered) enveloping algebra to the (graded) symmetric algebra, given by the natural projections

$$
\pi_{n}: \mathcal{U}^{(n)} \longrightarrow S^{n}=\mathcal{U}^{(n)} / \mathcal{U}^{(n-1)}
$$

and project (4.4) to the symmetric algebra (isomorphic to the polynomial algebra). It is obvious that the polynomial $\pi_{n}(4 C+1)$ is not the square of another polynomial. It follows that (4.4) cannot be satisfied unless $B_{0}=$ $B_{1}=0$.

\section{Theorem 4.5.}

$$
\mathcal{D}_{m}=\mathcal{U} /\left(C-\frac{m}{2}\left(\frac{m}{2}+1\right) \mathrm{Id}\right) .
$$


Proof. Immediate from the definition of $\mathcal{D}$ and the Lemma 4.4.

We now want to make an explicit comparison with the result of deformation quantization, let us make the rescaling

$$
\tilde{X} \mapsto \hbar X, \quad \tilde{Y} \mapsto \hbar Y, \quad \tilde{H} \mapsto \hbar H, \quad \tilde{D}=\hbar D .
$$

In what follows, $\hbar$ is a number, not an indeterminate; so we are comparing the geometric quantization with the specialization for a value of $\hbar$ of the deformation of the polynomial algebra obtained in Section 3. Notice that with this rescaling we obtain a family of isomorphic Lie algebras

$$
[\tilde{H}, \tilde{X}]=\hbar 2 \tilde{X}, \quad[\tilde{H}, \tilde{Y}]=-\hbar 2 \tilde{Y}, \quad[\tilde{X}, \tilde{Y}]=\hbar \tilde{H}
$$

(and $\tilde{D}$ in the center) except for $\hbar \mapsto 0$ (while keeping the generators constant) in which the algebra becomes abelian. $\mathcal{U}_{\hbar}$ is the enveloping algebra of the Lie algebra for each value of $\hbar$.

The Casimir operator is

$$
\tilde{C}=\frac{1}{2}\left(\tilde{X} \tilde{Y}+\tilde{Y} \tilde{X}+\frac{1}{2} \tilde{H}^{2}\right) .
$$

Using (4.4), the corresponding ideal in $\mathcal{U}_{\hbar}$ is

$$
(\tilde{C}-l(l+\hbar)), \quad l=\hbar m / 2 .
$$

It is enough to take $c(\hbar)=l(l+\hbar)$ to obtain the result of Section 3 .

Since $l$ is the eigenvalue of the central element $D / 2$ in the corresponding representation, taking the limit $\hbar \mapsto 0$ and keeping the generators constant (abelian Lie algebra) is equivalent to take $m \mapsto \infty$. In the physical picture one says that the classical limit corresponds to large quantum numbers.

We want to make the following observations. By choosing different polynomials $c(h)$ and different values of $h$ we obtain that the specialized $\mathbf{C}$ algebras in general are not isomorphic. In fact, it is a known result (see [Va1]) that $\mathcal{U} /(C-\mu 1)$ has no finite dimensional representations when $\mu$ is not rational, hence different values of $\mu$ (that is of $c(h)$ ) may give nonisomorphic algebras.

We also want to remark that our deformation quantization not only gives a subalgebra that can be specialized for any value of $h$ (namely the subalgebra of elements that have coefficients that are polynomials in $h$ ), but in the special case of $S U(2), S L(2, \mathbf{C})$, when $h$ is taking certain values, realizes the subalgebra as a concrete algebra of differential operators on the space of sections described above.

Finally, comparing with the approach of $[\mathbf{B B E W}]$, it is easy to see that the subalgebra of observables with converging star product is the same as the one we obtain, that is, the algebra of polynomials on the algebraic manifold. 
Acknowledgements. We wish to thank especially Prof. Varadarajan for his help during the preparation of this paper. We also wish to thank A. Brown, S. Ferrara, D. Gieseker and G. Mess for helpful discussions.

\section{References}

[ACG] D. Arnal, M. Cahen and S. Gutt, Deformations on coadjoint orbits, J. Geom. Phys., 3 (1987), 327-351.

[ALM] D. Arnal, J. Ludwig and M. Masmoudi, Déformations covariantes sur les orbites polarisées d'un group de Lie, J. Geom. Phys., 14 (1994), 309-331.

[BFFLS] F. Bayen, M. Flato, C. Fronsdal, A. Lichnerowicz and D. Sternheimer, Deformation theory and quantization, I and II, Ann. Phys., 111(1) (1978), 61-151.

[Be] F.A. Berezin, Quantization, Math. USSR Izvestija, 8 (1974), 1109-1165.

[BBEW] M. Bordemann, M. Brischle, C. Emmrich and S. Waldmann, Subalgebras with converging star products in deformation quantization, preprint q-alg/9512019, (1995).

[Bo] A. Borel, Linear Algebraic Groups, Springer Verlag, (1991).

[C] B. Cahen, Deformation program for principal series representations, Lett. Math. Phys., 36 (1996), 65-75.

[CG] M. Cahen and S. Gutt, Produits * sur les orbites des groupes semi-simples de rang 1, C.R. Acad. Sc. Paris, 296 (1983), série I, 821-823; An algebraic construction of $*$ product on the regular orbits of semisimple Lie groups, in 'Gravitation and Cosmology', Monographs and Textbooks in Physical Sciences. A volume in honor of Ivor Robunian, Bibliopolis, Eds W. Rundler and A. Trautman, (1987); Non-localité d'une déformation symplectique sur la sphère $S^{2}$, Bull. Soc. Math. Belg., 36 B (1987), 207-221.

[CGR] M. Cahen, S. Gutt and J. Rawnsley, On tangential star product for the coadjoint Poisson Structure, Comm. Math. Phys., 180 (1996), 99-108.

[CP] V. Chari and A. Pressley, A Guide to Quantum Groups, Cambridge University Press, (1994).

[DL] M. De Wilde and P.B.A. Lecomte, Existence of star-products and of formal deformations in Poisson Lie algebras of arbitrary symplectic manifolds, Lett. Math. Phys., 7 (1983), 487-496.

[EK] P. Etingof and D.A. Kazhdan, Quantization of Lie bialgebras, I, Selecta Math., New Series 2(1) (1996), 1-41.

[Fe] B. Fedosov, A simple geometric construction of deformation quantization, J. Diff. Geom., 40(2) (1994), 213-238.

[GV] J.M. García Bondía and Joseph C. Varilly, From geometric quantization to Moyal quantization, J. Math. Phys., 36 (1995), 2691-2701.

[Gu] S. Gutt, An explicit *-product on the cotangent bundle of a Lie group, Lett. Math. Phys., 7 (1983), 249-258.

[HC] Harish-Chandra, Representations of a semisimple Lie group I, II, II, Trans. Amer. Math. Soc., 75 (1953), 185-243; 76 (1954), 26-65; 76 (1954), 234-253. 
[Ho] J. Hoppe, On the deformation of time harmonic flows, in 'Deformation Theory and Symplectic Geometry', proceedings of the Ascona meeting, June 1996, Eds. D. Sternheimer, J. Rawnsley and S. Gutt, Kluwer Academic Publishers, (1997).

[Ki] A. Kirillov, Elements of the Theory of Representations, Springer Verlag, (1975).

[Ko] M. Kontsevich, Deformation Quantization of Poisson Manifolds, preprint qalg/9709040, (1997).

[Ks] B. Kostant, Lie group representations on polynomial rings, Amer. J. of Math., 86 (1964), 271-309.

$[\mathrm{Pu}] \quad$ M. Puta, Hamiltonian Mechanical Systems and Geometric Quantization, Kluwer Academic Publishers, (1993).

[RCG] J.Rawsnsley, M. Cahen and S. Gutt, Quantization of Kähler manifolds I: Geometric interpretation of Berezin's quantization, J. Geom. Phys., 7(1) (1990), 45-62.

[RT] N. Reshetekhin and L. Takhtajan, Deformation Quantization of Kähler Manifolds, preprint, (1999).

[So] Sorieau, Structure des Systémes Dynamiques, Dunod, Paris, (1970).

[Va1] V.S. Varadarajan, Lie Groups, Lie Algebras and their Representations, Springer Verlag, (1984).

[Va2] Harmonic Analysis on Real Reductive Groups, Lecture Notes in Mathematics, 576, Springer-Verlag, (1977).

[Va3] - On the ring of invariant polynomials on a semisimple Lie algebra, Amer. J. Math, 90 (1968), 308-317.

[Vo] D. Vogan, The Orbit Method and Unitary Representations for Reductive Lie Groups, in 'Algebraic and Analytic Methods in Representation Theory', Perspectives in Mathematics, Vol. 17, Academic Press, (1996).

Received June 2, 1999. Supported by EEC under TMR contract ERBFMRX-CT96-0045, (Politecnico di Torino).

Universita Di Bologna

Piazza Porta San Donato 5

40125 BolOGNA

ITALY

E-mail address: fioresi@dm.unibo.it

Dipartimento Di FisicA

PoliteCnico Di Torino

Corso Duca degli Abruzzi, 24

10129 TORINO

ITALY

Istituto Nazionale di Fisica Nucleare (INFN)

SEzione di Torino

ITALY

E-mail address: lledo@athena.polito.it 